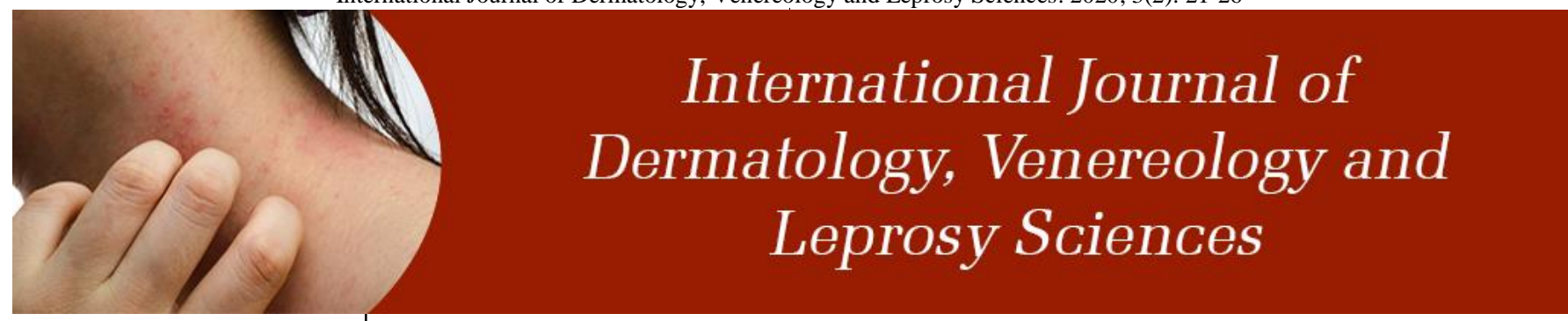

E-ISSN: 2664-942X

P-ISSN: 2664-9411

www.dermatologypaper.com

Derma 2020; 3(2): 21-26

Received: 28-04-2020

Accepted: 16-06-2020

Sukmawati Tansil Tan

Departement of Dermato-

Venerology, Tarumanagara

University, Jakarta, Indonesia

Yohanes Firmansyah

Departement of Dermato-

Venerology, Tarumanagara

University, Jakarta, Indonesia

Jessica Elizabeth

Departement of Dermato-

Venerology, Tarumanagara

University, Jakarta, Indonesia

\section{New approach to deep diabetic foot ulcer (DFU) treatment-potential of secretome from Wharton's jelly mesenchymal stem cell therapy}

\author{
Sukmawati Tansil Tan, Yohanes Firmansyah and Jessica Elizabeth
}

DOI: https://doi.org/10.33545/26649411.2020.v3.i2a.41

\begin{abstract}
Diabetic foot ulcer is one of the complications of chronic diabetes in the form of lesions in deep tissue and associated neurological disorders and peripheral vascular disease in the lower limbs. DFU has a significant impact on the health and quality of life of patients and their families. The effect that is often felt is ranging from pain, loss of function and mobility, depression, difficulty and anxiety, shame, social isolation, the financial burden of extended hospital stays and chronic morbidity, or even amputation to death. This case report reports the case of a 64-year-old man with DFU grade 3 and has been advised to undergo a finger amputation surgery by a surgeon to prevent infection, and the finger is predicted to be untenable. After intervention with a single dose, intracutaneous injection of Secretome from-Placental Wharton Jelly Stem Cell (SC-PWJSC), regular insulin, and control Sugar Blood Glucose. There is a very significant wound repair. Patients reported being satisfied with this treatment.
\end{abstract}

Keywords: Diabetic foot ulcer, diabetes mellitus, cutaneous injection, SC-PWJSC

\section{Introduction \\ 1. Background}

Diabetic foot ulcer is one of the complications of chronic diabetes in the form of lesions in deep tissue and associated neurological disorders and peripheral vascular disease in the lower limbs ${ }^{[1]}$. The incidence of diabetic foot ulcers has increased due to the prevalence of diabetes mellitus worldwide and the better life expectancy of diabetic patients. Literature searches show that every 30 seconds, an amputation occurs in the lower extremities due to diabetes ${ }^{[2]}$, and caused a significant health cost burden of $\$ 8659$ per patient ${ }^{[3]}$. Total medical costs for treating diabetic foot disease in the United States range from $\$ 9$ to $\$ 13$ billion and are additional costs associated with diabetes ${ }^{[4]}$. The International Diabetes Foundation has appealed to the public to increase awareness of diabetic foot disease due to a substantial social, medical, and economic burden ${ }^{[5]}$. Of all amputations in diabetic patients, $85 \%$ are preceded by foot ulceration which then worsens into severe gangrene or infection which is difficult to resolve ${ }^{[6]}$.

The prevalence of diabetic foot ulcers globally reaches $6.3 \%$ (95\% CI: $5.4-7.3 \%)$, which is higher in men $(4.5 \%, 95 \%$ CI: $3.7-5.2 \%)$ compared to women $(3.5 \%, 95 \%$ CI: $2.8-4.2 \%)$, and higher in patients with type 2 diabetes $(6.4 \%, 95 \%$ CI: 4.6-8.1\%) compared to people with type 1 diabetes $(5.5 \%, 95 \%$ CI: $3.2-7.7 \%)$. North America has the highest prevalence (13.0\%, 95\% CI: $10.0-15.9 \%)$, Oceania has the lowest $(3.0 \%, 95 \%$ CI: $0.9-5.0 \%)$, and prevalence in Asia, Europe, and Africa respectively 5.5\% (95\% CI: 4.6-6.4\%), 5.1\% (95\% CI: $4.1-6.0 \%)$, and $7.2 \%$ (95\% CI: 5.1-9.3\%). Australia has the lowest prevalence (1.5\%, 95\% CI: $0.7-2.4 \%)$ and Belgium has the highest prevalence $(16.6 \%, 95 \%$ CI: $10.7-22.4 \%)$, followed by Canada (14.8\%, 95\% CI: $9.4-20.1 \%)$ and the US $(13.0 \%, 95 \%$ CI: $8.3-17.7 \%)$. (6) In 2016, the World Health Organization noted the prevalence of diabetes in Indonesia was $7 \%$ of the total population. Since 1980, the prevalence of diabetes in Indonesia has continued to increase. The percentage of diabetic ulcers as a complication of diabetes mellitus in 2011 at RSUP Dr. Cipto Mangunkusumo Jakarta (RSCM) is $8.70 \%$. ${ }^{[7]}$.

The rate of wound healing in chronic wounds, especially Diabetic foot ulcers (DFU), is very low ${ }^{[8]}$. has a significant impact on the health and quality of life of patients and their families. The effect that is often felt is ranging from pain, loss of function and mobility, depression,
Corresponding Author: Sukmawati Tansil Tan Departement of DermatoVenerology, Tarumanagara University, Jakarta, Indonesia 
difficulty and anxiety, shame, social isolation, the financial burden of extended hospital stays and chronic morbidity or even death ${ }^{[9]}$. Previous studies have proven that chronic ulcers are a significant burden and often make the quality of life of their sufferers very poor. Therefore, it is essential to perform wound care and management of chronic ulcers that are efficient and inexpensive to improve the quality of life of people with diabetic foot ulcers ${ }^{[8,10,11]}$

This case report explains that the potential use of single dose intracutaneous injection of the secretome from Placental Wharton Jelly Stem Cell (SC-PWJSC) to patients with diabetic foot in the lower extremities. Diabetes foot care in these patients follows the applicable diabetes foot management protocol with the addition of Secretome from Placental Wharton Jelly Stem Cell (SC-PWJSC) an additional therapy to trigger better-wound healing or we suggest as a gold standard therapy in wound management.

\section{Case Report}

A 64-year-old man came with complaints of wounds that have not healed in the right big toe since the last year and have worsened over the past seven months. Patients have been advised to undergo finger amputation surgery by a surgeon to prevent infection, and the finger is predicted to be untenable. Patients visit to start alternative therapies using single dose intracutaneous injection of secretome from Placental Wharton Jelly Stem Cell (SC-PWJSC)

Past medical history was found that patients have had type 2 diabetes mellitus for more than ten years. Sugar levels are not well controlled with Glibenklamid treatment. History of high blood pressure is denied; the history of stroke is denied. The patient denies a history of haematology disorder, denied heart and kidney diseases.

The patient current subjective complaint is discomfort in the legs accompanied by pain, delayed chronic wound healing, discharge and unpleasant odor form the wound. The patient also complained of numbness in the toes, especially in the right big toe. Patients routinely use Povidone iodine to clean the wound to prevent infection and unpleasant odors.

On physical examination found a grade 3 diabetes ulcer. The neovascular status around the wound is a blackish blue wound with CRT prolonged in the wound and cold areas accompanied by local anesthesia in the wound area. (Figures 1 and 2)

Patients signed up the agreement to follow treatment using single dose intracutaneous injection of secretome from Placental Wharton Jelly Stem Cell (SC-PWJSC) and control routinely for two weeks. Patients were also given secretome gel from Placental Wharton Jelly Stem Cell (SC-PWJSC) to be applied every day after the wound was cleaned with $\mathrm{NaCl}$. Patients are also asked to note the symptoms of side effects that may arise from allergic reactions such as itching, redness, burning sensation, and swelling to seek first aid if severe side effects appear that are very disturbing.

The patient came back to control one week later; granulation tissue began to appear, the bluish color was reduced, dead skin tissue was whitish around the wound, visible dead tissue in the wound pushed out of the wound hole. The patient's neovascular status also improved from the first day of visit with the color around the bluish-red wound, but anesthesia still occurred. Patients reported being satisfied with development for one week in the absence of significant side effects. (Figures 3 and 4)

The patient came back to control after three weeks of intervention with a closed wound accompanied by scarring without secondary side effects. Symptoms of side effects were not found during the intervention. Treatment during the intervention is the only treatment with injection of insulin and secretome gel from Placental Wharton Jelly Stem Cell (SC-PWJSC) accompanied by regular blood sugar control for which the regulated dose of diabetes mellitus is treated. (Figures 5 and 6)

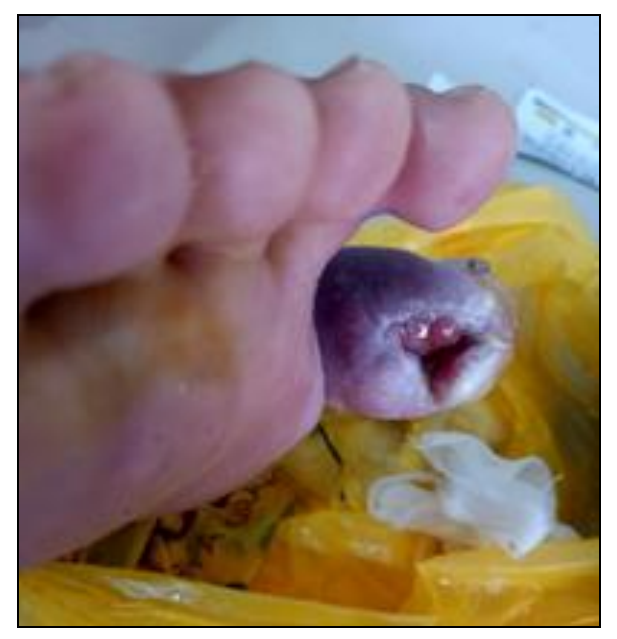

Fig 1: A grade 3 diabetes foot ulcer

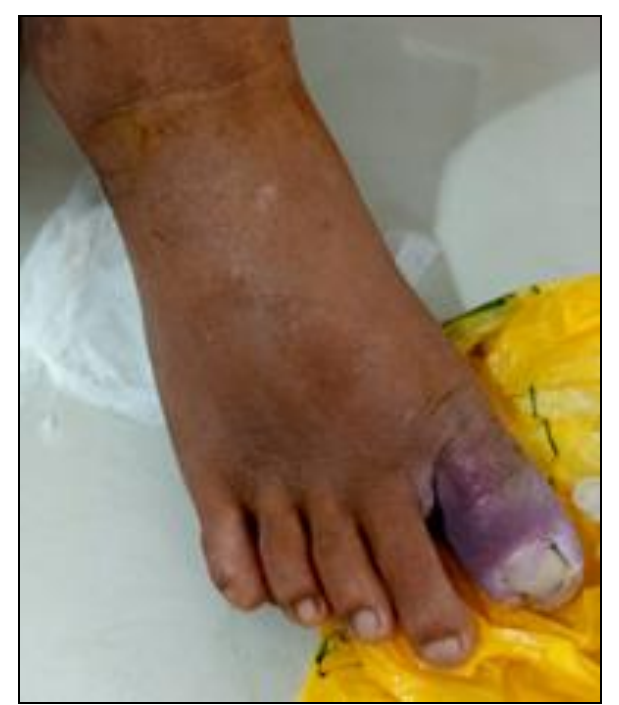

Fig 2: The neovascular status around the wound is a blackish blue with prolonged CRT and cold areas accompanied by local anesthesia in the wound area

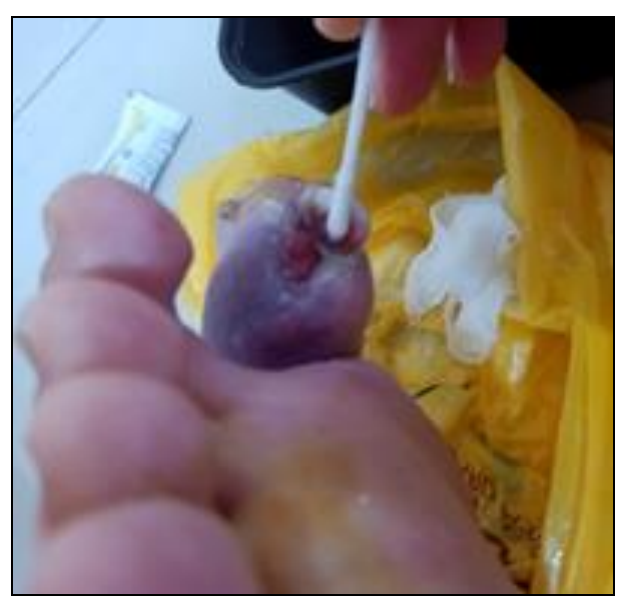

Fig 3: The bluish color was reduced 


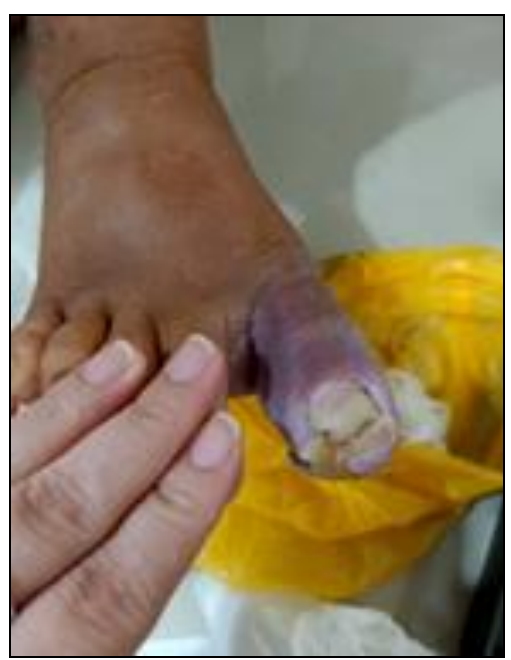

Fig 4: Granulation tissue began to appear; the bluish color was reduced, dead skin tissue was whitish around the wound, visible dead tissue in the wound pushed out of the wound hole

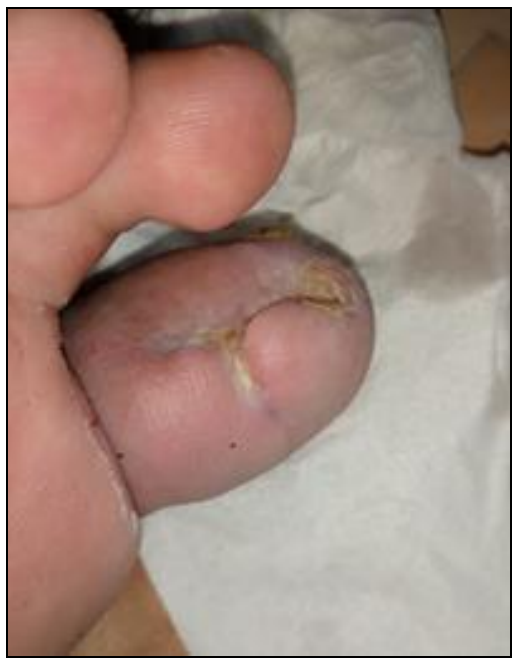

Fig 5: A closed wound accompanied by scarring

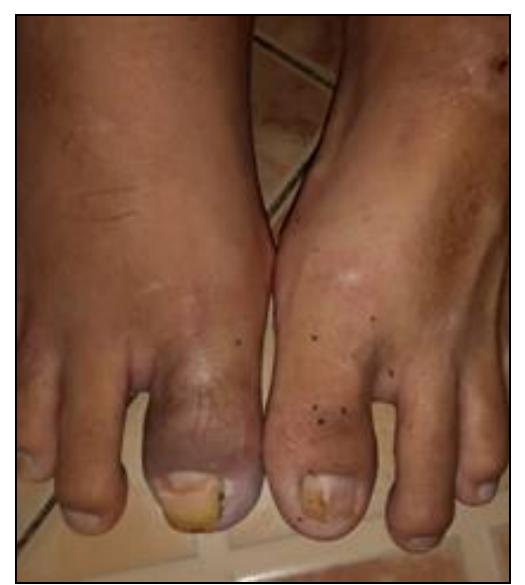

Fig 6: A closed wound accompanied by scarring

\section{Discussion}

Stem cell therapy is a new therapy that brings new hope to new wound healing methods and is following the physiology of wound healing in general. These stem cells contain growth and healing factors to repair wounds ${ }^{[12]}$. This wound healing process through the process of withdrawing cells that are not differentiated into the matrix by healing factors and induce cell division to close the wound. On the other hand, these stem cells also play a role in immunomodulation through cytokine suppression, suppressing inflammation, interacting with macrophage regulators. This whole process impacts on tissue regeneration, new capillary growth (angiogenesis) and accelerates the process of epithelialization in chronic wounds so that healing occurs as before ${ }^{[13,14]}$.

One form of stem cells is Mesenchymal stem cells (MSCs), which can be isolated or obtained from various types of original tissue ${ }^{[15]}$. In general, mesenchymal stem cells (MSCs) can be harvested from placental tissue and its derivatives such as umbilical cord, newborn skin, and placenta ${ }^{[16,17]}$. Planting or using Mesenchymal stem cells (MSCs) in damaged tissue sites (locations of chronic ulcers) will support angiogenesis and vasculogenesis while modulating the immune system. This whole process plays a vital role in the process of wound healing, especially chronic ulcers (Figure 7) ${ }^{[18]}$.

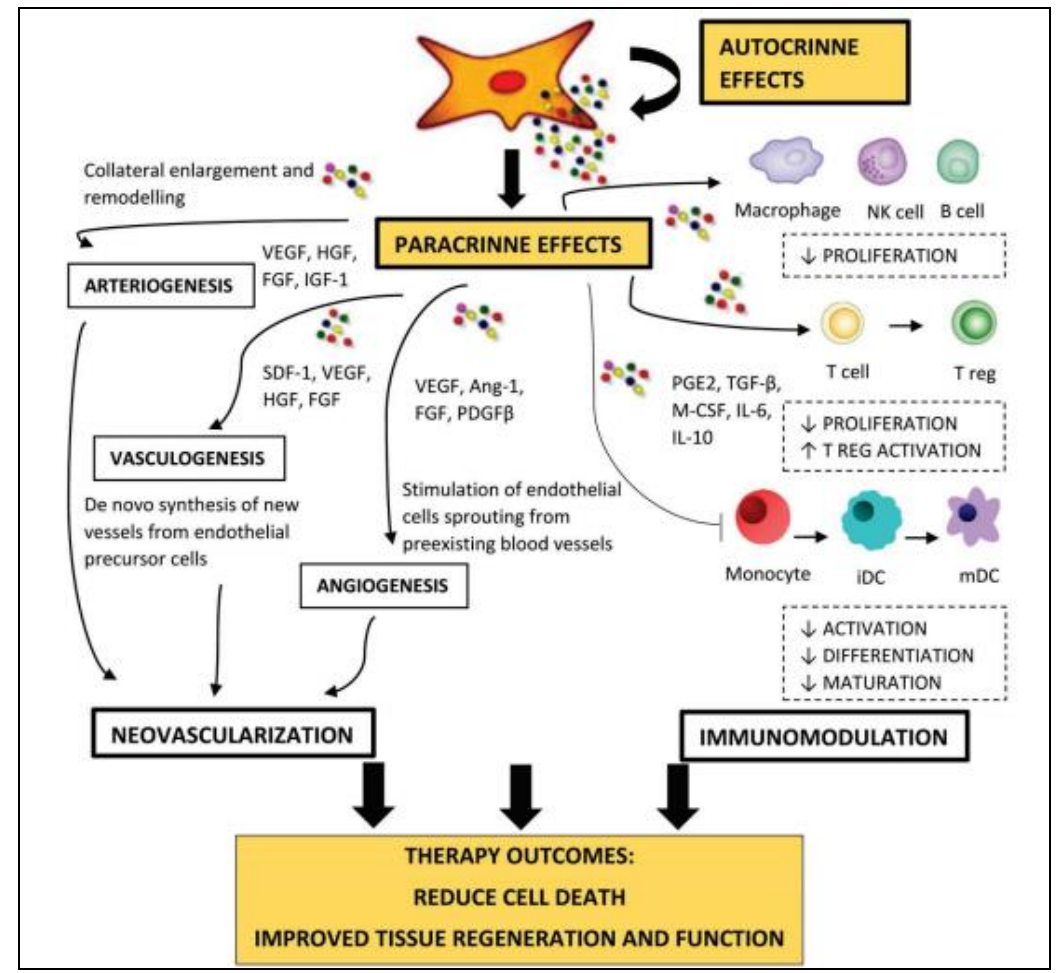

Fig 7: Mechanisms of Angiogenesis and Vasculogenesis in the Treatment of Chronic Ulcers [19] 
The process of wound healing using Mesenchymal stem cells (MSCs) is a process that resembles the physiology of wound healing in general, which starts from Mesenchymal stem cells (MSCs) will differentiate into fibroblast and pericytes tissue and form endothelial-like tissue or cells which further play a role in vasculogenesis ${ }^{[20,21]}$. Previous literature researches reveal that Wharton's Jelly derived mesenchymal stem cells (WJ-MSCs) have a rich composition of undifferentiated cells that have excellent nontumorigenic and immunomodulatory properties and do not cause rejection reactions in the allotransplantation process in repairing organs major such as heart, cartilage, liver, bone, pancreas, fat components, blood vessels to the skin. ${ }^{(22)}$ MSCs produced from WJ-MSCs in the extracellular space release several angiogenic factors including basal fibroblast growth factor (bFGF) and VEGF which alter betagrowth factor (TGF- $\beta$ ), PDGF, ANG-1, placental growth factor (PIGF), IL -6, hepatocyte growth factor (HGF), and monocyte one chemoattractant protein (MCP-1), which stimulates angiogenesis in vitro and in vivo ${ }^{[22]}$. VEGF and TGF- $\beta 1$ secreted in CM promote angiogenesis and activate the PI3K/Akt and MAPK pathways; HGF shows its angiogenic nature by inducing VEGF expression ${ }^{[19]}$.

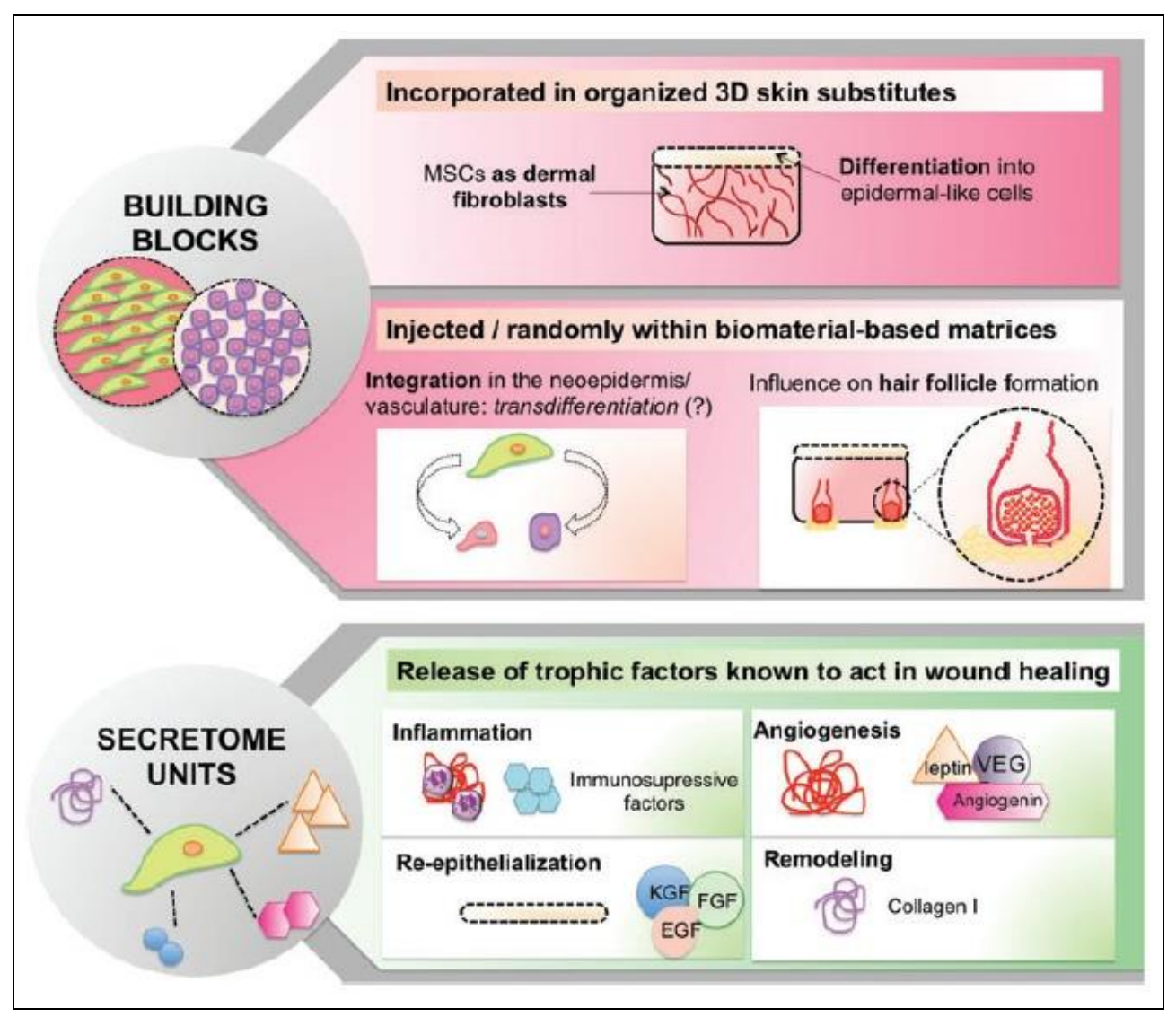

Fig 8: The schematic perspective of stem cells in skin wound healing and as potent secretome units at different response levels ${ }^{(23)}$

The use of MSC which is rich in secretome topically in the field of regenerative medicine has several primary advantages from the provision of stem cells by other methods, namely

1. The use of secretomes has excellent safety compared to live cell transplantation directly (invasive).

2. Secretors originating from MSC is always evaluated for its safety, dosage, and potential.

3. Topical MSC has a function or efficacy that is no less effective than invasive administration, including modulating the immune system, angiogenesis, and wound healing.

4. Easy storage and can last for a long time without decreasing efficacy or even being toxic.

5. Secretome originating from Wharton's Jelly-derived mesenchymal stem cell (WJ-MSCs) umbilical cord has economic and practical value and can be massproduced under standard laboratory control.

6. Umbilical cord Wharton's Jelly derived mesenchymal stem cells (WJ-MSCs) can not only be used for healing chronic ulcers but also can be used for various other medical fields such as acute wounds, cerebral ischemia and myocardial.

7. It is flexible and can be used as a therapeutic application and can be modified for various types of cells as desired ${ }^{[23]}$.

Conditioned Media (CM) is an ideal environment and has a variety of elements that play a role in storing various regeneration factors of cell tissue and tissue. Dissolved components consisting of microvesicles and secretomes can be separated singly by multiple methods. The method that can be used to divide it in the form of microvesicle fraction consisting of various stages, namely centrifugation, filtration, polymer-based deposition methods, ion exchange chromatography, and size-based chromatography. All elements in Conditioned Media (CM) related to tissue repair and regeneration independently and induced the occurrence of organogenesis de novo and ex vivo tissue ${ }^{[24]}$. 


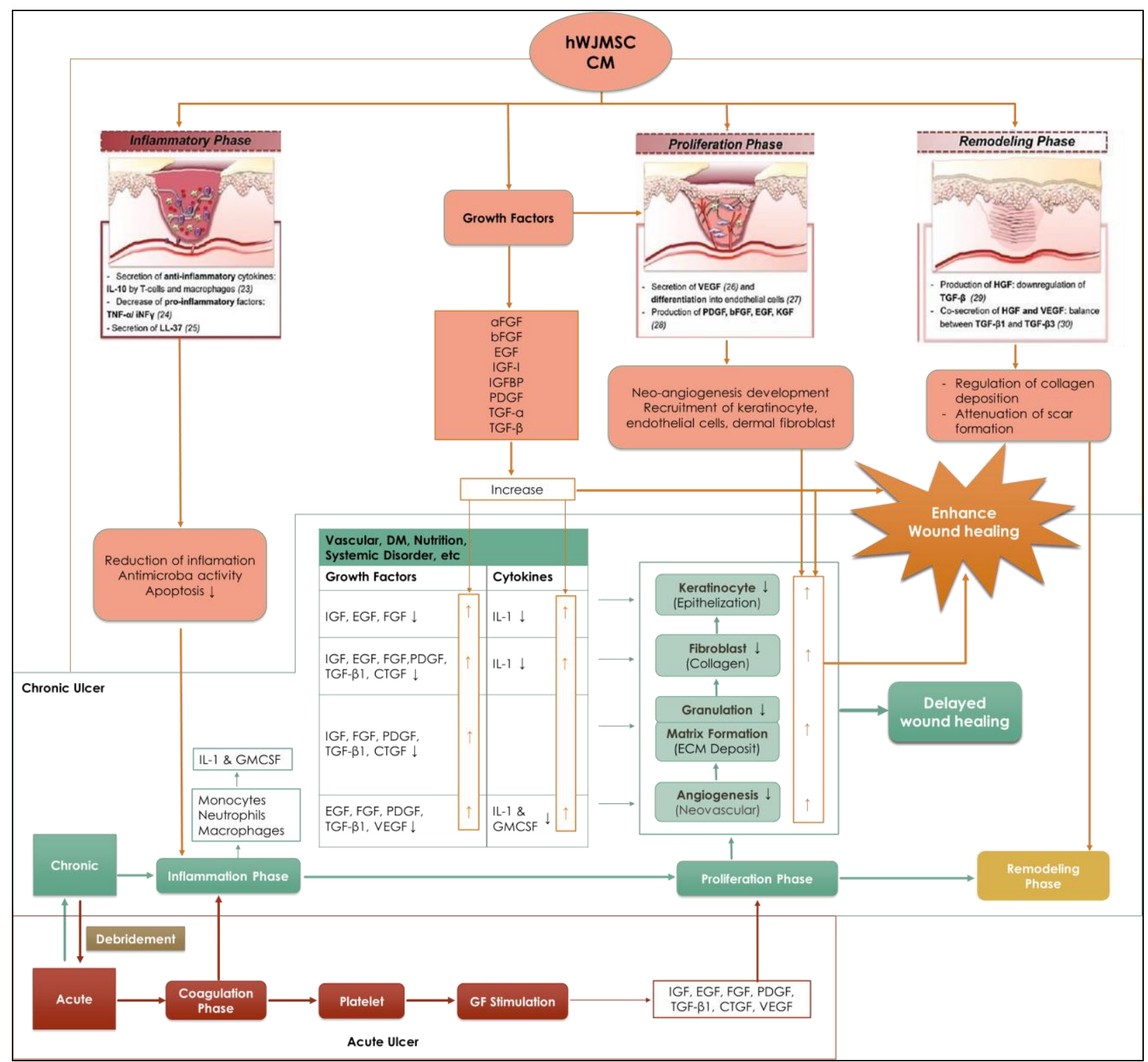

Fig 9: This scheme shows the summary of Mechanism-secretome from Placental Wharton Jelly Stem Cell (SC-PWJSC) in enhancing diabetic wound healing in every step of the wound healing process

This case report demonstrates the excellent expectation result without side effects by using a single dose intracutaneous injection of the secretome from Placental Wharton Jelly Stem Cell (SC-PWJSC) therapy that is very easy and can even be applied independently to patients with promising outcomes and reduces future disability. In the future, this research can be carried out on a large scale in Indonesia, starting with serial case reports, clinical trials, and randomized controlled trials (RCTs).

\section{Conclusion}

Diabetic foot ulcer is one of the complications of chronic diabetes in the form of lesions in deep tissue and associated neurological disorders and peripheral vascular disease in the lower limbs. DFU has a significant impact on the health and quality of life of patients and their families. The effect that is often felt is ranging from pain, loss of function and mobility, depression, difficulty and anxiety, shame, social isolation, the financial burden of extended hospital stays and chronic morbidity, or even death. One DFU case was reported in a 64 -year-old man who came with complaints of wounds that have not healed in the right big toe since the last year and have worsened over the past seven months.
Patients have been advised to undergo finger amputation surgery by a surgeon to prevent infection, and the finger is predicted to be untenable. The intervention was carried out in the form of the wound injected with a single dose intracutaneous injection of the secretome from Placental Wharton Jelly Stem Cell (SC-PWJSC) around the wound. Patients were also given SC-PWJSC gel to be applied every day after the wound was cleaned with $\mathrm{NaCl}$. After three weeks of intervention with a closed wound accompanied by scarring without secondary side effects. Patients reported being satisfied with this treatment.

\section{Reference}

1. Apelqvist J. Diagnostics and treatment of the diabetic foot. Endocrine, 2012.

2. Boulton AJ, Vileikyte L, Ragnarson-Tennvall G, Apelqvist $\mathrm{J}$ The global burden of diabetic foot disease. Lancet, 2005.

3. Ragnarson Tennvall G, Apelqvist J. Health-Economic Consequences of Diabetic Foot Lesions. Clin Infect Dis, 2004.

4. Rice JB, Desai U, Cummings AKG, Birnbaum HG, Skornicki M, Parsons NB. Burden of diabetic foot 
ulcers for medicare and private insurers. Diabetes Care, 2014.

5. Jeffcoate W, Bakker K. World Diabetes Day: footing the bill. Lancet [Internet]. 2005; 365(9470):1527. Available from: https://linkinghub.elsevier.com/retrieve/pii/S014067360 5664379

6. Zhang P, Lu J, Jing Y, Tang S, Zhu D, Bi Y. Global epidemiology of diabetic foot ulceration: a systematic review and meta-analysis. Annals of Medicine, 2017.

7. Soelistijo SA, Novida H, Rudijanto A, Soewondo P, Suastika K, Manaf A et al. Konsensus Pengendalian dan Pencegahan Diabetes Melitus Tipe 2 di Indonesia. Perkeni, 2015.

8. Järbrink K, Ni G, Sönnergren H, Schmidtchen A, Pang C, Bajpai R et al. Prevalence and incidence of chronic wounds and related complications: A protocol for a systematic review. Syst Rev, 2016.

9. Macdonald J. Global Initiative for Wound and Lymphoedema Care (GIWLC). Wounds UK, 2009.

10. Denny K, Lawand C, Perry SD. Compromised wounds in Canada. Healthc Q, 2014.

11. Heyer K, Augustin M, Protz K, Herberger K, Spehr C, Rustenbach SJ. Effectiveness of advanced versus conventional wound dressings on healing of chronic wounds: Systematic review and meta-analysis. Dermatology, 2013.

12. Bluestein D, Javaheri A. Pressure ulcers: Prevention, evaluation, and management. American Family Physician, 2008.

13. Bielecki T, S Gazdzik T, Arendt J, Szczepanski T, Król W, Wielkoszyński T. Antibacterial effect of autologous platelet gel enriched with growth factors and other active substances: An in vitro study. The Journal of bone and joint surgery. British, 2007; 89:417-420.

14. Abrigo M, McArthur SL, Kingshott P. Electrospun nanofibers as dressings for chronic wound care: advances, challenges, and future prospects. Macromol Biosci. 2014; 14(6):772-92.

15. Pittenger MF, Mackay AM, Beck SC, Jaiswal RK, Douglas R, Mosca JD et al. Multilineage potential of adult human mesenchymal stem cells. Science (80-). 1999; 284(5411):143-7.

16. Kim SM, Lim JY, Park SI, Jeong CH, Oh JH, Jeong M, et al. Gene therapy using TRAIL-secreting human umbilical cord blood-derived mesenchymal stem cells against intracranial glioma. Cancer Res. 2008; 68(23):9614-23.

17. Arno AI, Amini-Nik S, Blit PH, Al-Shehab M, Belo C, Herer E et al. Human Wharton's jelly mesenchymal stem cells promote skin wound healing through paracrine signaling. Stem Cell Res Ther, 2014.

18. Klopp AH, Gupta A, Spaeth E, Andreeff M, Marini F. Concise review: Dissecting a discrepancy in the literature: Do mesenchymal stem cells support or suppress tumor growth? Stem Cells, 2011.

19. Samakova A, Gazova A, Sabova N, Valaskova S, Jurikova M, Kyselovic J. The pi3k/Akt pathway is associated with angiogenesis, oxidative stress and survival of mesenchymal stem cells in pathophysiologic condition in ischemia. Physiol Res, 2019.

20. Spaeth EL, Dembinski JL, Sasser AK, Watson K, Klopp A, Hall B et al. Mesenchymal stem cell transition to tumor-associated fibroblasts contributes to fibrovascular network expansion and tumor progression. PLoS One. 2009; 4(4):e4992.

21. Short B, Brouard N, Occhiodoro-Scott T, Ramakrishnan A, Simmons PJ. Mesenchymal stem cells. Arch Med Res. 2003; 34(6):565-71.

22. Nekanti U, Rao VB, Bahirvani AG, Jan M, Totey S, Ta M. Long-Term Expansion and Pluripotent Marker Array Analysis of Wharton's Jelly-Derived Mesenchymal Stem Cells. Stem Cells Dev. 2010; 19(1):117-30.

23. Cerqueira MT, Pirraco RP, Marques AP. Stem cells in skin wound healing: are we there yet? Adv Wound Care. 2016; 5(4):164-75.

24. Vizoso FJ, Eiro N, Cid S, Schneider J, Perez-Fernandez R. Mesenchymal stem cell secretome: Toward cell-free therapeutic strategies in regenerative medicine. International Journal of Molecular Sciences, 2017. 